\title{
AN ANTIBODY WHICH SUBDIVIDES THE HUMAN MN BLOOD GROUPS
}

\author{
RUTH SANGER * and R. R. RACE \\ Medical Research Council Blood Group Research Unit, Lister Institute, London, S.W. I \\ and \\ R. J. WALSH and CARMEL MONTGOMERY \\ New South Wales Blood Transfusion Service, Sydney
}

Received 8.viii.47

AN antibody has been found in a sample of human serum which subdivides the $\mathrm{MN}$ blood groups, as we have already shown (1947).

The donor of the serum containing this agglutinin was a woman whose fifth pregnancy had just terminated in the delivery of a stillborn macerated foetus. She had never been transfused, but had had one normal child followed by a second which died, aged one day, of erythroblastosis fotalis; the third and fourth pregnancies had ended in early miscarriages. The blood groups of the family are as follows :-

\section{Mother : A cde/cde MN \\ Father: A GDe/CDe M \\ Child : A GDe/cde}

The serum contains anti-D of the "incomplete" type, which undoubtedly was responsible for the disease of the two infants, but it also contains the agglutinin whose reactions are the subject of this paper.

The following table shows the results of agglutination tests on blood from Igo unselected English people, the tests were carried out in saline and at room temperature. (The agglutinin is also active at $37^{\circ} \mathrm{C}$.)

\begin{tabular}{|c|c|c|c|c|c|c|}
\hline \multicolumn{2}{|c|}{$\mathbf{M}$} & \multicolumn{2}{|c|}{$\mathrm{MN}$} & \multicolumn{2}{|c|}{$\mathbf{N}$} & Total \\
\hline+ & - & + & - & + & - & $\ldots$ \\
\hline 36 & 14 & 57 & 38 & 15 & 30 & 190 \\
\hline $18.95 \%$ & $7.37 \%$ & $30 \cdot 00 \%$ & $20 \cdot 00 \%$ & $7 \cdot 89 \%$ & $15 \cdot 79 \%$ & $100 \cdot 00 \%$ \\
\hline
\end{tabular}

* Supported by the Australian Red Cross. 
The association of the "new" antibody with the MN system is clear from the following $2 \times 2$ table :-

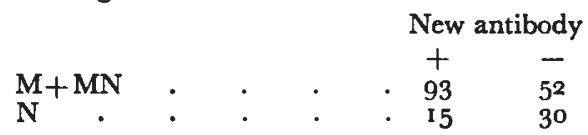

for which the $\chi^{2}$ equals I 3 for I d.f., corresponding to a probability of less than o.0or. No significant association with the $\mathrm{A}_{1} \mathrm{~A}_{2} \mathrm{BO}$ groups, nor the $\mathrm{P}$ groups, nor any of the $\mathrm{Rh}$ antigens can be found. The antibody does not give the reactions of the rare agglutinins " anti-Lutheran," " anti-Kell " nor " anti-Lewis."

A reasonable genetical interpretation is that there are four allelomorphs at the locus responsible for these groups $\mathrm{M}, \mathrm{MS}, \mathrm{N}$ and NS, the mutation $S$ being a change which can happen both to $M$ and to $\mathrm{N}$ genes, and which makes the resulting red cells agglutinable by the new antibody.

On the other hand, it is possible that $\mathrm{S}$ is a separate linked gene, presumably having an allelomorph not $S$ (or s). If an antibody corresponding to not $S$ were found, this interpretation of a separate locus would seem the more probable. Anti- not $S$ would agglutinate about 88 per cent. of English bloods. The situation would then be very similar to that of the $\mathrm{C}, \mathrm{D}$ and $\mathrm{E}$ antigens of the $\mathrm{Rh}$ system. If the interpretation of linked genes is correct, the linkage must be very close, otherwise crossing over would presumably have resulted in an equilibrium in which the ratio of MS to $M$ would equal that of NS to $\mathrm{N}$.

The calculations given below lend strong support to one or other of these genetical interpretations of the observed reactions.

The simple postulation of a third gene allelomorphic to $\mathrm{M}$ and $\mathrm{N}$, say $\mathrm{L}$, will not meet the facts, for $\mathrm{MN}$ blood is frequently agglutinated by the new antibody, and the presence of three allelomorphs in one person would have to be invoked.

\section{GENE FREQUENCIES}

In the calculations shown below both the symbols and the figures will fit the idea of 4 allelomorphs or of separate but closely linked genes.

\begin{tabular}{|c|c|c|c|c|c|c|}
\hline \multicolumn{2}{|c|}{$\mathbf{M}$} & \multicolumn{2}{|c|}{ MN } & \multicolumn{2}{|c|}{$\mathbf{N}$} & Total \\
\hline $\begin{array}{c}{ }^{+} \\
\mathrm{MS} \text { MS }\end{array}$ & $\overline{\mathrm{MM}}$ & $\begin{array}{c}+ \\
\text { MS N } \\
\text { MS NS } \\
\text { M NS }\end{array}$ & $\overline{\mathrm{MN}}$ & $\begin{array}{c}+ \\
\text { NS N } \\
\text { NS NS }\end{array}$ & $\overline{\mathrm{NN}}$ & \\
\hline $\begin{array}{c}3^{6} \\
0 \cdot{ }^{8995}\end{array}$ & $\begin{array}{c}14 \\
0.0737\end{array}$ & $\begin{array}{c}57 \\
0.3000\end{array}$ & $\begin{array}{c}3^{8} \\
0 \cdot 2000\end{array}$ & $\begin{array}{c}15 \\
0 \cdot 0789\end{array}$ & $\begin{array}{c}30 \\
0 \cdot 1579\end{array}$ & $\begin{array}{c}\text { r } 90 \\
\text { I.0000 }\end{array}$ \\
\hline
\end{tabular}


We are greatly indebted to Professor Fisher who has estimated the gene frequencies by his method of maximum likelihood (1946), with the following results as percentages :-

\begin{tabular}{|c|c|c|c|c|}
\hline MS & • & . & • & $25 \cdot 0487$ \\
\hline $\mathbf{M}$ & • & . & $\boldsymbol{\theta}^{-}$ & $26 \cdot 267$ \\
\hline NS & - & ${ }^{\circ}$ & . & $9 \cdot 2$ I I I \\
\hline$N$. & - & . & . & $39 \cdot 472$ I \\
\hline
\end{tabular}

These gene frequencies can be used to calculate the expected frequencies of bloods in the various phenotype groups.

\begin{tabular}{|c|c|c|c|}
\hline & Expected & Observed & $x^{2}$ \\
\hline \multirow[t]{2}{*}{$\left.\begin{array}{l}\text { MS MS } \\
\text { MS M } \\
\text { MM } \\
\text { MS N } \\
\text { MS NS } \\
\text { M NS }\end{array}\right\}$} & $\begin{array}{l}36 \cdot 9236 \\
13 \cdot 1093 \\
55 \cdot 5351 \\
39 \cdot 3991 \\
15 \cdot 4300 \\
29 \cdot 6029\end{array}$ & $\begin{array}{l}36 \\
14 \\
57 \\
38 \\
15 \\
30\end{array}$ & $\begin{array}{l}0 \cdot 02310 \\
0 \cdot 06052 \\
0 \cdot 03864 \\
0 \cdot 04970 \\
0 \cdot 01198 \\
0 \cdot 00533\end{array}$ \\
\hline & $190 \cdot 0000$ & 190 & $\begin{array}{c}0 \cdot 18927 \\
\text { d.f. } 2 \\
p=0.9\end{array}$ \\
\hline
\end{tabular}

The agreement between expected and observed frequencies is seen to be very close.

\section{FAMILY INVESTIGATIONS}

The subdivisions of the MN groups defined by this serum greatly increase the number of distinguishable matings. There are only 6 phenotypically and genotypically distinct types of classical MN matings, but with the help of the new antibody there are theoretically 2 I phenotypically and 55 genotypically different matings. A list of these matings is given in table $\mathrm{I}$, with the approximate frequencies of their occurrence.

The results of the family investigations are given in table 2. In the second column of this table the classification of the type of mating refers to the catalogue of matings given in table $I$. Sometimes the exact type of mating is known as in families $3,4,6,9,13,14,20,22,23,24$, $25,26,27,29$ and 30 . Sometimes only the main group to which the mating belongs can be recognised. Sometimes the main group is known but one of the constituent matings can be excluded; for example, family I 5 belongs to type I 3 , but cannot be I $_{3} a$ for the latter mating does not produce MN children. 


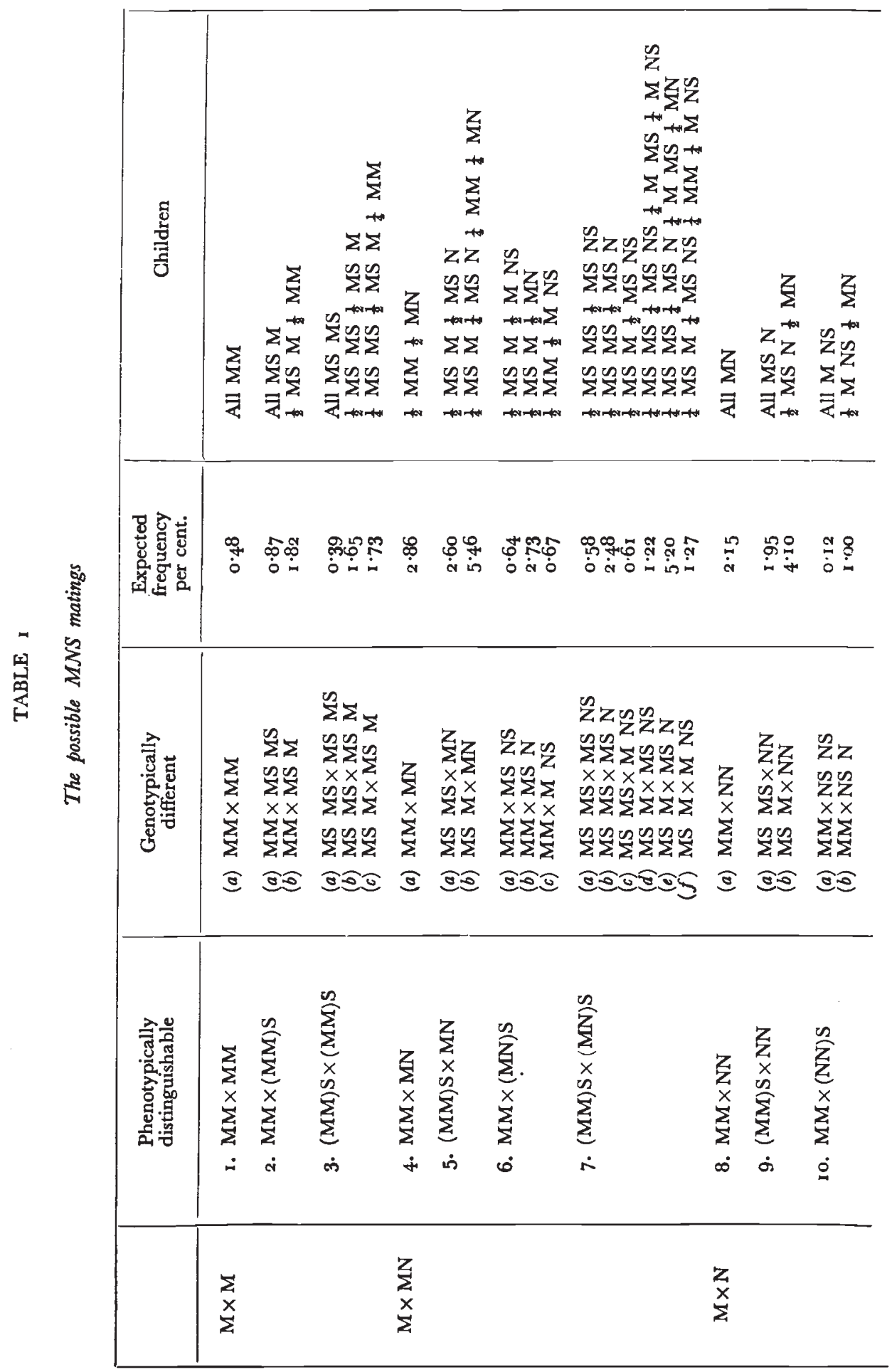




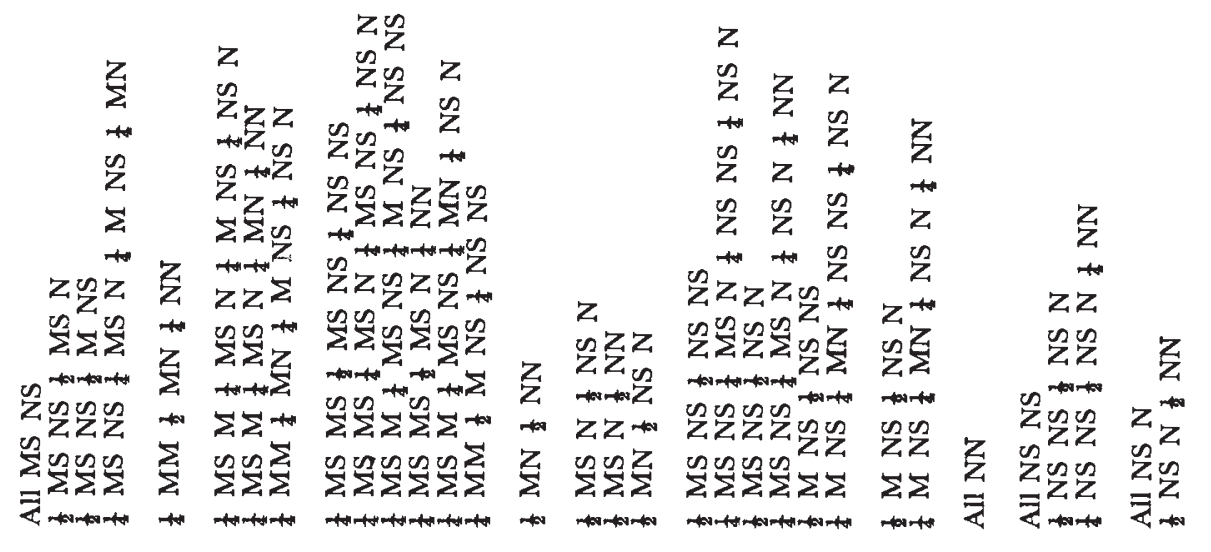

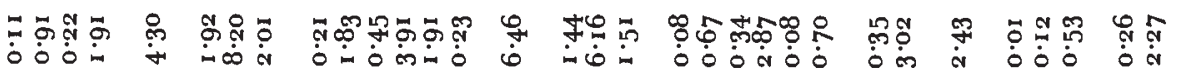

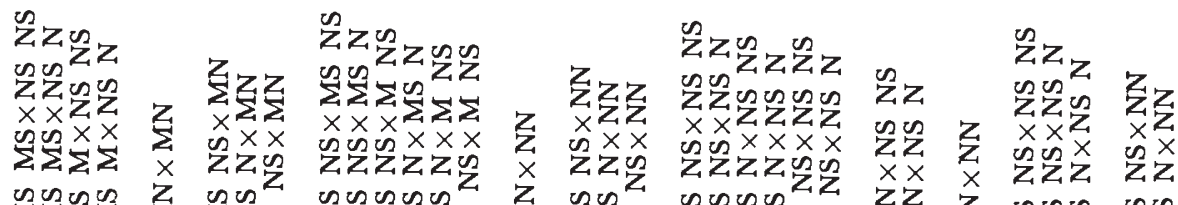

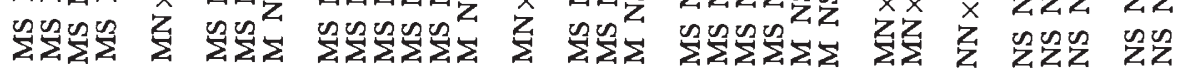

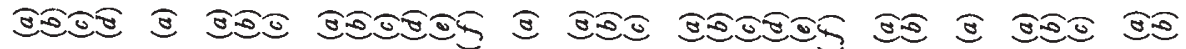

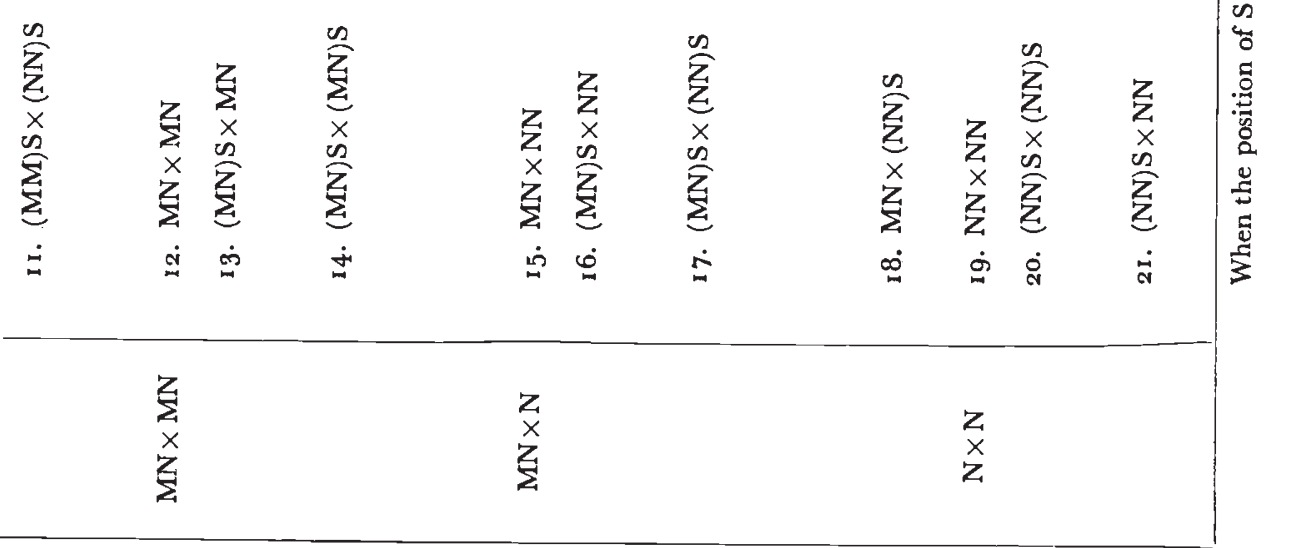


TABLE 2

The results of family investigations

\begin{tabular}{|c|c|c|c|c|c|c|c|c|c|c|}
\hline \multirow{2}{*}{ No. } & \multirow{2}{*}{$\begin{array}{c}\text { Type } \\
\text { of } \\
\text { Mating }\end{array}$} & \multicolumn{2}{|c|}{ Parents } & \multicolumn{7}{|c|}{ Children } \\
\hline & & Father & Mother & 1 & 2 & 3 & 4 & 5 & 6 & 7 \\
\hline $\mathbf{I}^{*}$ & 2 & $\begin{array}{c}\mathrm{MM} \\
\mathbf{B} \\
\mathbf{R}_{2} \mathbf{R}_{2}\end{array}$ & $\begin{array}{c}(\mathbf{M M}) \mathrm{S} \\
\mathbf{A}_{1} \\
\mathbf{R}_{1} \mathbf{R}_{2}\end{array}$ & $\begin{array}{c}\text { MS M } \\
\mathrm{R}_{\mathbf{2}} \mathrm{R}_{\mathbf{2}}\end{array}$ & $\begin{array}{c}\mathrm{MS} \mathbf{M} \\
\mathrm{R}_{2} \mathrm{R}_{\mathbf{2}}\end{array}$ & $\begin{array}{c}\mathrm{MS} \mathrm{M} \\
\mathbf{A}_{1} \mathbf{B} \\
\mathrm{R}_{1} \mathbf{R}_{2}\end{array}$ & & & & \\
\hline 2 & 3 & $\begin{array}{c}(\mathbf{M M}) \mathbf{S} \\
\mathbf{A} \\
\mathrm{R}_{1} \mathbf{R}_{0}\end{array}$ & $\begin{array}{c}(\mathrm{MM}) \mathrm{S} \\
\mathrm{A} \\
r r\end{array}$ & $\begin{array}{c}(\mathrm{MM}) \mathrm{S} \\
\mathbf{A} \\
\mathrm{R}_{0} r\end{array}$ & $\begin{array}{c}(\mathbf{M M}) \mathbf{S} \\
\mathbf{A} \\
\mathbf{R}_{0} r\end{array}$ & & & & & \\
\hline 3 & $6 b$ & $\begin{array}{c}\mathrm{MS} N \\
A \\
R_{1} R_{1}\end{array}$ & $\begin{array}{c}\mathrm{MM} \\
\mathrm{R}^{\prime \prime} r\end{array}$ & $\begin{array}{c}\text { MS M } \\
\mathrm{R}_{1} \mathbf{R}^{\prime \prime}\end{array}$ & $\begin{array}{c}\mathrm{MN} \\
\mathrm{O} \\
\mathrm{R}_{\mathbf{1}} \mathrm{R}^{\prime \prime}\end{array}$ & $\begin{array}{c}\mathrm{MN} \\
\mathrm{A}_{1} \\
\mathrm{R}_{1} r\end{array}$ & $\begin{array}{c}\mathrm{MN} \\
\mathrm{A} \\
\mathrm{R}_{1}{ }^{r}\end{array}$ & $\begin{array}{c}\mathbf{M S} \mathbf{M} \\
\mathbf{R}_{\mathbf{1}} \mathbf{R}^{\prime \prime}\end{array}$ & $\begin{array}{c}\mathrm{MN} \\
\mathrm{R}_{1} \mathrm{R}^{\prime \prime}\end{array}$ & $\begin{array}{c}\mathrm{MS} \mathbf{M} \\
\mathrm{O}_{\mathbf{1}} \mathbf{R}^{\prime \prime}\end{array}$ \\
\hline $4 \dagger$ & $6 b$ & $\begin{array}{c}\mathrm{MM} \\
\mathbf{A}_{\mathbf{2}} \\
\mathrm{R}_{\mathbf{1}} \mathbf{R}_{\mathbf{1}}\end{array}$ & $\begin{array}{c}\mathrm{MS} N \\
\mathrm{R}_{1^{\top}}\end{array}$ & $\begin{array}{c}(\mathbf{M M}) \mathbf{S} \\
\mathbf{A}_{\mathbf{i}} \\
\mathbf{R}_{\mathbf{1}^{r}}\end{array}$ & $\begin{array}{c}\mathrm{MN} \\
\mathrm{O} \\
\mathrm{R}_{1} \mathrm{R}_{1}\end{array}$ & $\begin{array}{c}\mathrm{MN} \\
\mathrm{O} \\
\mathrm{R}_{1} r\end{array}$ & $\begin{array}{c}\mathrm{MN} \\
\mathbf{A}_{\mathbf{2}} \\
\mathrm{R}_{\mathbf{1}} \mathbf{R}_{1}\end{array}$ & & & \\
\hline 5 & 5 & $\begin{array}{c}\text { (MM)S } \\
\mathrm{O} \\
\mathrm{R}_{1}{ }^{\mathrm{w}} \mathrm{R}^{\prime} \\
\text { Lewis- } \\
\text { Kell+ } \\
\mathbf{P}\end{array}$ & $\begin{array}{c}\mathrm{MN} \\
\mathrm{A}_{1} \\
\mathrm{R}_{1}{ }^{r} \\
\text { Lewis+ } \\
\text { Kell - } \\
\mathbf{P}\end{array}$ & $\begin{array}{c}\text { MS N } \\
\text { O } \\
R^{\prime} r \\
\text { Lewis }+ \\
\text { Kell - } \\
\text { P }\end{array}$ & $\begin{array}{c}\text { MS M } \\
\mathbf{A}_{\mathbf{1}} \\
\mathbf{R}^{\prime} r \\
\text { Lewis }+ \\
\text { Kell }+ \\
\mathbf{P}\end{array}$ & $\begin{array}{c}\text { MS N } \\
\mathbf{A}_{1} \\
\mathbf{R}_{1} \mathbf{R}^{\prime} \\
\text { Lewis }+ \\
\text { Kell+ } \\
\mathbf{P}\end{array}$ & & & & \\
\hline 6 & $7 e$ & $\begin{array}{c}\mathrm{MS} M \\
\mathrm{R}_{1} \mathrm{R}_{1}\end{array}$ & $\begin{array}{c}\mathrm{MS} N \\
\mathbb{R}_{1} \mathrm{R}_{1}\end{array}$ & $\begin{array}{c}(\mathrm{MM}) \mathrm{S} \\
\mathrm{O} \\
\mathbf{R}_{1} \mathbf{R}_{1}\end{array}$ & $\underset{\mathrm{R}_{1} \mathbf{R}_{1}}{\mathrm{MN}}$ & $\begin{array}{c}(\mathbf{M M}) \mathrm{S} \\
\mathrm{O} \\
\mathrm{R}_{1} \mathbf{R}_{1}\end{array}$ & & & & \\
\hline 7 & 7 & $\begin{array}{c}(\mathrm{MN}) \mathrm{S} \\
\mathrm{A}_{1} \mathrm{~B} \\
\mathrm{R}_{1} \mathbf{R}_{1}\end{array}$ & $\begin{array}{c}(\mathbf{M M}) \mathrm{S} \\
\mathrm{O} \\
\mathbf{R}_{\mathbf{1}} \mathbf{R}_{\mathbf{2}}\end{array}$ & $\begin{array}{c}(\mathbf{M M}) \mathrm{S} \\
\mathbf{B} \\
\mathrm{R}_{1} \mathbf{R}_{1}\end{array}$ & & & & & & \\
\hline 8 & 7 & $\begin{array}{c}(\mathrm{MN}) \mathrm{S} \\
\mathrm{A} \\
\mathrm{R}_{1} \mathbf{R}_{1}\end{array}$ & $\underset{\mathbf{R}_{2} r}{(\mathbf{M M}) \mathrm{S}}$ & $\begin{array}{c}(\mathrm{MM}) \mathrm{S} \\
\mathrm{O} \\
\mathrm{R}_{1} \mathrm{R}_{2}\end{array}$ & $\begin{array}{c}(\mathbf{M M}) \mathbf{S} \\
\mathbf{A} \\
\mathbf{R}_{\mathbf{1}} \mathbf{R}_{\mathbf{8}}\end{array}$ & & & & & \\
\hline 9 & $9^{b}$ & $\begin{array}{c}\mathrm{MS} \mathbf{M} \\
\mathrm{R}_{\mathbf{2}} \mathbf{R}_{\mathbf{2}}\end{array}$ & $\begin{array}{c}\mathrm{NN} \\
\mathrm{O} \\
\mathrm{R}_{2} r\end{array}$ & $\begin{array}{c}\text { MS N } \\
O \\
\mathrm{R}_{2} \mathbf{R}_{2}\end{array}$ & $\begin{array}{c}\mathrm{MN} \\
\mathrm{O} \\
\mathbf{R}_{\mathbf{2}} r\end{array}$ & $\begin{array}{c}\mathrm{MN} \\
\mathrm{O} \\
\mathrm{R}_{2} r\end{array}$ & & & & \\
\hline 10 & 9 & $\begin{array}{c}\mathrm{NN} \\
\mathrm{O} \\
\mathrm{R}_{1} r\end{array}$ & $\begin{array}{c}(\mathrm{MM}) \mathrm{S} \\
\mathrm{O} \\
r r\end{array}$ & $\begin{array}{c}\text { MS N } \\
\mathrm{O} \\
r r\end{array}$ & & & & & & \\
\hline 11 & II & $\begin{array}{c}(\mathrm{NN}) \mathrm{S} \\
\mathrm{O} \\
\mathrm{R}_{\mathbf{1}^{r}}\end{array}$ & $\begin{array}{c}(\mathbf{M M}) \mathbf{S} \\
\mathbf{A} \\
\mathbf{R}_{1} \mathbf{R}_{2}\end{array}$ & $\begin{array}{c}(\mathrm{MN}) \mathrm{S} \\
\mathbf{A} \\
\mathrm{R}_{\mathbf{2}} r\end{array}$ & $\begin{array}{c}(\mathrm{MN}) \mathrm{S} \\
\mathbf{A} \\
\mathbf{R}_{1} r\end{array}$ & $\begin{array}{c}(\mathbf{M N}) \mathbf{S} \\
\mathbf{A} \\
\mathbf{R}_{\mathbf{1}} \mathbf{R}_{\mathbf{2}}\end{array}$ & & & & \\
\hline 12 & II & $\begin{array}{c}(\mathrm{NN}) \mathrm{S} \\
\mathrm{O} \\
\mathrm{R}_{\mathbf{1}}{ }^{r}\end{array}$ & $\begin{array}{c}(\mathbf{M M}) \mathrm{S} \\
\mathbf{B} \\
\mathbf{R}_{\mathbf{1}^{r}}\end{array}$ & $\begin{array}{c}(\mathrm{MN}) \mathrm{S} \\
\mathrm{O} \\
\mathrm{R}_{\mathbf{1}^{r}}\end{array}$ & 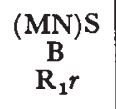 & $\begin{array}{c}(\mathrm{MN}) \mathrm{S} \\
\mathrm{O} \\
\mathrm{R}_{\mathbf{1}^{r}}\end{array}$ & & & & \\
\hline 13 & $12 a$ & $\begin{array}{c}\mathrm{MN} \\
\mathrm{O} \\
\mathrm{R}_{1} r\end{array}$ & $\begin{array}{c}\mathrm{MN} \\
\mathrm{O} \\
r r\end{array}$ & $\underset{r r}{\mathrm{MN}}$ & $\stackrel{\mathrm{MN}}{\mathrm{O}}$ & $\begin{array}{c}\mathrm{MN} \\
\mathrm{O}\end{array}$ & & & & \\
\hline 14 & $12 a$ & $\begin{array}{c}\mathrm{MN} \\
\mathbf{A}_{1} \\
\mathbf{R}_{1} r\end{array}$ & $\begin{array}{l}\mathrm{MN} \\
\mathrm{A}_{1} \\
\mathrm{R}_{1} r\end{array}$ & $\begin{array}{c}\mathrm{MN} \\
\mathbf{A}_{1} \\
\mathrm{R}_{\mathbf{1}} \mathbf{R}_{\mathbf{1}}\end{array}$ & & & & & & \\
\hline 15 & $\begin{array}{c}13 \\
\text { not } a\end{array}$ & $\begin{array}{c}(\mathrm{MN}) \mathrm{S} \\
\mathrm{O} \\
\mathrm{R}_{\mathbf{1}} \mathrm{R}_{1}\end{array}$ & $\begin{array}{c}\mathbf{M N} \\
\mathbf{O} \\
\mathbf{R}_{2} \mathbf{R}_{2}\end{array}$ & $\begin{array}{c}\mathrm{MN} \\
\mathrm{O} \\
\mathrm{R}_{1} \mathrm{R}_{2}\end{array}$ & $\begin{array}{c}(\mathbf{M N}) \mathrm{S} \\
\mathrm{O} \\
\mathbf{R}_{\mathbf{1}} \mathbf{R}_{\mathbf{2}}\end{array}$ & $\begin{array}{c}(\mathrm{MN}) \mathrm{S} \\
\mathrm{O} \\
\mathrm{R}_{1} \mathrm{R}_{\mathbf{8}}\end{array}$ & & & & \\
\hline 16 & $\begin{array}{c}14 \\
\text { not } d\end{array}$ & $\begin{array}{c}(\mathrm{MN}) \mathrm{S} \\
\mathrm{A}_{1} \mathrm{~B} \\
\mathrm{R}_{1}{ }^{r}\end{array}$ & $\underset{r}{(\mathrm{MN}) \mathrm{S}}$ & $\begin{array}{c}\left(\mathrm{MN}_{\mathrm{B}}\right) \mathrm{S} \\
\mathrm{R}_{1}{ }^{r}\end{array}$ & $\begin{array}{c}(\mathrm{NN}) \mathrm{S} \\
\mathrm{B} \\
r r\end{array}$ & & & & & \\
\hline
\end{tabular}

* Ist child is father in family 9. 2nd child is mother in family 21. $\dagger$ ist child by previous husband. 


\begin{tabular}{|c|c|c|c|c|c|c|c|c|c|c|}
\hline \multirow{2}{*}{ No. } & \multirow{2}{*}{$\begin{array}{c}\text { Type } \\
\text { of } \\
\text { Mating }\end{array}$} & \multicolumn{2}{|c|}{ Parents } & \multicolumn{7}{|c|}{ Children } \\
\hline & & Father & Mother & I & 2 & 3 & 4 & 5 & 6 & 7 \\
\hline 17 & I 14 & $\begin{array}{c}(\mathrm{MN}) \mathrm{S} \\
\mathrm{A}_{1} \mathrm{~B}^{\mathrm{B}} \\
\mathrm{R}_{\mathbf{3}} \tau\end{array}$ & $\begin{array}{c}(\mathrm{MN}) \mathrm{S} \\
\mathrm{O} \\
\pi\end{array}$ & $\begin{array}{c}(\mathrm{MM}) \mathrm{S} \\
\mathrm{B} \\
\mathrm{R}_{2} r\end{array}$ & $\begin{array}{c}(\mathrm{NN}) \mathrm{S} \\
\mathrm{A}_{1} \\
r r\end{array}$ & & & & & \\
\hline $18+$ & 14 & $\begin{array}{c}(\mathbf{M N}) \mathrm{S} \\
\mathbf{A}_{1} \\
\mathbf{R}_{1}{ }^{r}\end{array}$ & $\begin{array}{c}(\mathrm{MN}) \mathrm{S} \\
\mathrm{B} \\
\mathbf{R}_{\mathbf{1}} \mathbf{R}_{\mathbf{2}}\end{array}$ & $\begin{array}{c}(\mathrm{MN}) \mathrm{S} \\
\mathrm{A}_{1} \mathrm{~B} \\
\mathrm{R}_{\mathbf{1}} \mathbf{R}_{\mathbf{1}}\end{array}$ & $\begin{array}{c}(\mathrm{MN}) \mathrm{S} \\
\mathbf{A}_{\mathbf{1}} \mathbf{B} \\
\mathbf{R}_{\mathbf{1}^{r}}\end{array}$ & $\begin{array}{c}(\mathbf{M N}) \mathrm{S} \\
\mathbf{A}_{\mathbf{1}} \mathbf{B} \\
\mathbf{R}_{\mathbf{2}} r\end{array}$ & & & & \\
\hline 19 & ${ }_{\text {not }}^{\mathrm{I}}$ & $\begin{array}{c}(\mathrm{MN}) \mathrm{S} \\
\mathbf{A}_{1} \\
\mathbf{R}_{2} r\end{array}$ & $\begin{array}{c}(\mathrm{MN}) \mathrm{S} \\
\mathbf{A} \\
\mathbf{R}_{\mathbf{1}} r\end{array}$ & $\begin{array}{c}(\mathbf{M N}) \mathrm{S} \\
\mathbf{A}_{1} \\
\mathbf{R}_{2} r\end{array}$ & $\begin{array}{c}(\mathbf{M M}) \mathrm{S} \\
\mathbf{A} \\
\mathbf{R}_{1} r\end{array}$ & & & & & \\
\hline 20 & $12 a$ & $\begin{array}{c}\mathrm{MN} \\
\mathrm{O} \\
\mathrm{R}_{\mathbf{1}} \mathrm{R}_{1}\end{array}$ & $\begin{array}{c}\mathrm{MN} \\
\mathrm{A}_{1} \\
\mathrm{R}_{1}{ }^{w_{r}}\end{array}$ & $\begin{array}{c}\mathrm{MN} \\
\mathrm{O} \\
\mathrm{R}_{1} r\end{array}$ & $\begin{array}{c}\mathrm{MN} \\
\mathrm{O} \\
\mathrm{R}_{1}{ }^{\mathrm{w}} \mathrm{R}_{1}\end{array}$ & $\stackrel{\stackrel{M N}{O}}{\stackrel{\mathrm{O}}{\mathrm{R}_{1}{ }^{\mathrm{w}} \mathrm{R}_{1}}}$ & $\begin{array}{c}\mathrm{MM} \\
\mathbf{A}_{1} \\
\mathrm{R}_{1} r\end{array}$ & & & \\
\hline $21 \xi$ & $\mid \begin{array}{l}7 d \\
e, r\end{array}$ & $\begin{array}{c}(\mathrm{MN}) \mathrm{S} \\
\mathrm{A}_{2} \\
\mathbf{R}_{2} r\end{array}$ & $\begin{array}{c}M \subseteq \mathrm{Ni} \\
\mathrm{O} \\
\mathrm{R}_{2} \mathrm{R}_{2}\end{array}$ & $\begin{array}{c}\text { MMiS } \\
O \\
\mathrm{R}_{2} r\end{array}$ & $\begin{array}{c}(\mathrm{MM}) \mathrm{S} \\
\mathrm{A}_{2} \\
\mathrm{R}_{2} \mathbf{R}_{2}\end{array}$ & & & & & \\
\hline 22 & $4^{4}$ & $\begin{array}{l}\mathrm{MU} \\
\mathrm{A}_{2} \\
\mathrm{~K}_{3}{ }^{\gamma}\end{array}$ & $\begin{array}{l}\text { MMI } \\
\mathbf{A}_{2} \\
\mathrm{R}_{1} r\end{array}$ & $\begin{array}{l}\mathrm{MN} \\
\mathrm{A}_{2} \\
r r\end{array}$ & $\underset{r r}{\mathrm{MM}}$ & & & & & \\
\hline 23 & $16 c$ & $\begin{array}{c}N N \\
O \\
R_{1} R_{2}\end{array}$ & $\begin{array}{c}M \underset{N}{\mathrm{~N} S} \\
\mathrm{R}_{2} r\end{array}$ & $\begin{array}{c}\mathrm{MN} \\
\mathrm{O} \\
\mathrm{R}_{1} r\end{array}$ & $\begin{array}{l}\mathrm{NSN} \\
\mathrm{O} \\
\mathrm{R}_{1} \mathrm{R}_{2}\end{array}$ & $\begin{array}{c}\mathrm{MN} \\
\mathrm{O} \\
\mathrm{R}_{1} r\end{array}$ & & & & \\
\hline 24 & $8 a$ & $\begin{array}{c}\mathrm{NN} \\
\mathbf{A}_{1} \\
\mathbf{R}_{1} \mathbf{R}_{1}\end{array}$ & $\begin{array}{c}\mathrm{MM} \\
\mathrm{O} \\
r r\end{array}$ & $\begin{array}{c}\mathrm{MN} \\
\mathrm{O} \\
\mathrm{R}_{1} r\end{array}$ & & & & & & \\
\hline 25 & $18 b$ & $\begin{array}{c}\mathrm{NS} \mathrm{N} \\
\mathrm{O} \\
\mathrm{R}_{1} r\end{array}$ & $\begin{array}{l}\mathrm{MN} \\
\mathrm{A}_{1} \\
\mathrm{R}_{1} r\end{array}$ & $\begin{array}{c}\mathrm{MN} \\
\mathrm{O} \\
\mathrm{R}_{1} r\end{array}$ & & & & & & \\
\hline 26 & I I $d$ & $\begin{array}{c}\text { MS M } \\
\mathrm{O} \\
r r \\
p p\end{array}$ & $\begin{array}{c}\text { NS N } \\
\mathbf{A}_{1} \\
\mathbf{R}_{2}^{r} \\
\mathbf{P}_{p}\end{array}$ & $\begin{array}{c}(\mathrm{MN}) \mathrm{S} \\
\mathbf{A}_{1} \\
\mathbf{R}_{2} r \\
p p\end{array}$ & $\begin{array}{c}\mathrm{MN} \\
\mathrm{O} \\
\mathrm{R}_{2} r \\
\mathrm{P} p\end{array}$ & $\begin{array}{c}(\mathrm{MN}) \mathrm{S} \\
\mathrm{A}_{1} \\
\mathbf{R}_{2} r \\
\mathbf{P}_{p}\end{array}$ & $\begin{array}{c}(\mathrm{MN}) \mathrm{S} \\
\mathrm{A}_{1} \\
r r \\
\mathrm{P} p\end{array}$ & & & \\
\hline 27 & $14 e$ & $\begin{array}{c}(\mathrm{MN}) \mathrm{S} \\
\mathbf{A}_{1} \\
\mathbf{R}_{\mathbf{2}} r\end{array}$ & $\begin{array}{c}(\mathrm{MN}) \mathrm{S} \\
\mathbf{O} \\
\mathrm{R}_{2} r\end{array}$ & $\begin{array}{c}\mathrm{MN} \\
\mathrm{A}_{1} \\
r r\end{array}$ & $\begin{array}{c}(\mathrm{MN}) \mathrm{S} \\
\mathbf{O} \\
\mathbf{R}_{\mathbf{2}} \mathbf{R}_{\mathbf{2}}\end{array}$ & $\begin{array}{c}\mathrm{MS} \mathrm{M} \\
\mathrm{O} \\
\mathrm{R}_{2} \mathrm{R}_{2}\end{array}$ & $\underset{\mathbf{O}) S}{(\mathrm{MN}}$ & & & \\
\hline 28 & 3 & $\begin{array}{c}(\mathbf{M M}) \mathrm{S} \\
\mathbf{O} \\
\mathbf{R}_{\mathbf{1}} \mathbf{R}_{\mathbf{2}} \\
\mathbf{P}\end{array}$ & $\begin{array}{c}(\mathrm{MM}) \mathrm{S} \\
\mathrm{O} \\
\mathbf{R}_{1} r \\
\mathbf{P}\end{array}$ & $\begin{array}{c}(\mathrm{MM}) \mathrm{S} \\
\mathrm{O} \\
\mathbf{R}_{\mathbf{1}} \mathbf{R}_{\mathbf{2}} \\
\mathbf{P}\end{array}$ & $\begin{array}{c}(\mathrm{MM}) \mathrm{S} \\
\mathbf{O} \\
\mathbf{R}_{\mathbf{1}} \mathbf{R}_{\mathbf{2}} \\
\mathbf{P}\end{array}$ & $\begin{array}{c}(\mathrm{MM}) \mathrm{S} \\
\mathbf{O} \\
\mathbf{R}_{\mathbf{1}} r \\
\mathbf{P}\end{array}$ & $\begin{array}{c}(\mathbf{M M}) \mathrm{S} \\
\mathbf{O} \\
\mathbf{R}_{1}{ }^{r} \\
\mathbf{P}\end{array}$ & $\begin{array}{c}(\mathbf{M M}) \mathrm{S} \\
\mathbf{O} \\
\mathbf{R}_{\mathbf{1}} \mathbf{R}_{\mathbf{2}} \\
\mathbf{P}\end{array}$ & & \\
\hline 29 & $4 a$ & $\begin{array}{c}\mathrm{MM} \\
\mathrm{O} \\
r r \\
p p\end{array}$ & $\begin{array}{c}\mathrm{MN} \\
\mathrm{O} \\
\mathrm{R}_{0} r \\
p p\end{array}$ & $\begin{array}{c}\mathrm{MM} \\
\mathrm{O} \\
\mathrm{R}_{0} r \\
p p\end{array}$ & $\begin{array}{l}\mathrm{MN} \\
\mathrm{O} \\
r r \\
p p\end{array}$ & $\begin{array}{c}\mathrm{MN} \\
\mathrm{O} \\
r r \\
p p\end{array}$ & $\begin{array}{c}\mathrm{MM} \\
\mathrm{O} \\
\mathrm{R}_{\mathbf{0}} r \\
p p\end{array}$ & $\begin{array}{c}\mathrm{MN} \\
\mathrm{O} \\
r r \\
p p\end{array}$ & & \\
\hline 30 & ${ }_{15} a$ & $\begin{array}{c}\mathrm{MN} \\
\mathrm{A}_{1} \\
r r \\
\mathrm{P} p\end{array}$ & $\begin{array}{l}\mathrm{NN} \\
\mathrm{B} \\
\mathrm{R}_{2} r \\
p p\end{array}$ & $\begin{array}{l}\mathrm{NN} \\
\mathrm{A}_{1} \\
\mathrm{R}_{2} r \\
\mathrm{P} p\end{array}$ & $\begin{array}{l}\mathrm{MN} \\
\mathrm{A}_{1} \\
r r \\
\mathrm{P} p\end{array}$ & $\begin{array}{l}\mathrm{NN} \\
\mathrm{A}_{2} \\
\mathrm{R}_{2} r \\
\mathrm{P} p\end{array}$ & $\begin{array}{c}\mathrm{MN} \\
\mathrm{A}_{1} \\
\mathrm{R}_{2} r \\
p p\end{array}$ & & & \\
\hline
\end{tabular}

(MN) S written thus indicates that the $\mathrm{S}$ may be located on either or both the chromosomes. When family grouping makes clear the position of the $S$, it is written thus, MS N or M NS or MS NS.

The short $\mathrm{Rh}$ symbols used in this table have the following significance :- $\mathrm{R}_{\mathbf{1}}=\mathrm{CD} e, \mathrm{R}_{\mathbf{2}}=c \mathrm{DE}$, $r=c d e, \mathrm{R}_{1}{ }^{w}=\mathrm{C}^{w} \mathrm{D} e, \mathrm{R}^{\prime}=\mathrm{C} d e, \mathrm{R}^{\prime \prime}=c d \mathrm{E}$ and $\mathrm{R}_{0}=c \mathrm{D} e$.

In the determination of the $\mathrm{Rh}$ genotypes the following antisera were used :-anti-C-C $\mathrm{C}^{w}-c-\mathrm{D}-\mathrm{E}-\mathrm{e}$.

$\ddagger$ Ist child is father of family 7 . 2nd child is father of family 16.3 rd child is father of family 17 . $\S$ Mother is and child in family 1 . 
Families 3,4 and 23 are of particular significance in the support of the association of $\mathrm{S}$ with the MN blood groups.

In family 3 , the father is $\mathrm{MN}$ and possesses $\mathrm{S}$ (that is to say, his blood is agglutinated by the new antibody), the mother is $M M$ and does not possess $S$. It will be seen that $S$ is segregating with the father's $M$; for the 3 children to whom he gave his $M$ all have $S$, whereas the 4 to whom he gave his $\mathrm{N}$ have not. The probability of this segregation being due to chance is $1 / 35$, using Fisher's exact method for $2 \times 2$ tables ; or 1 in 64 , postulating, as in this case we may, the theoretical i : I ratio.

Family 4 is of the same type of mating, excluding the first child (the issue of a previous marriage of the mother) ; there are 3 children who have received their mother's $\mathrm{N}$, but not her $\mathrm{S}$. If these three children are added to those of family 3 , the probability of the segregation being due to chance is reduced to $\mathrm{I}$ in I20. Although the group of the father of the first child in family 4 is not known, this child has received $M$ from his mother and has $S$. If it is allowed that this $\mathrm{S}$ has come from the mother, then the probability is further reduced to $\mathrm{I}$ in 330 .

In family 23 we have been fortunate in finding an example of the converse type of mating, that is to say, $M N S \times N N$. Here we see dependent segregation of $\mathrm{N}$ and $\mathrm{S}$, for the two children who have received their mother's $M$ have not $S$, and the one child who has received her $\mathrm{N}$ has $\mathrm{S}$.

Through the finding reported in this paper a glimpse is obtained of the complexity of the MN groups. Since their discovery by Landsteiner and Levine in 1927, the MN groups remained, it seemed, singularly uncomplicated in comparison with the $\mathrm{ABO}$ and $\mathrm{Rh}$ groups. Whether this insight will have other than theoretical interest depends upon the success of attempts to reproduce the antibody at will in human beings or rabbits.

\section{SUMMARY}

The reactions of an antibody which subdivides the MN blood groups are described.

This antibody agglutinates 72 per cent. of $\mathrm{M}, 60$ per cent. of $\mathrm{MN}$ and 33 per cent. of $\mathrm{N}$ bloods; such bloods are designated by the addition of the symbol $\mathrm{S}$.

Statistical analysis and family groupings are consistent with the hypothesis that either there are 4 allelomorphs MS, M, NS and N, or that $\mathrm{S}$ is a gene closely linked to the $\mathrm{MN}$ locus. The gene frequencies calculated are MS $25^{\circ} 0$ per cent., $\mathrm{M}_{26} \cdot 3$ per cent., NS 9.2 per cent., and $\mathrm{N} 39.5$ per cent. 


\section{REFERENCES}

FISHER, R. A. 1946 .

The fitting of gene frequencies to data on rhesus reactions.

Ann. Eug. 13, 150 and 223.

LANDSTEINER, K., AND LEVINE, P. 1927.

P. Soc. Exp. Biol. Med. 24, 600 and 94 I.

SANGER, RUTH, AND RACE, R. R. 1947.

Subdivisions of the $\mathrm{MN}$ blood groups in man.

Nature 160 , 505 .

WALSH, R. J., AND MONTGOMERY, CARMEL. 1947.

A new human iso-agglutinin subdividing the MN blood groups.

Nature 160,504 . 